\title{
A CAVITY-LESS MICROMACHINED CAPACITIVE PRESSURE SENSOR FOR WIRELESS OPERATION IN LIQUID AMBIENT
}

\author{
K. Takahata ${ }^{1}$ and Y.B. Gianchandani ${ }^{2}$
}

${ }^{1}$ Department of Electrical and Computer Engineering, University of British Columbia, Vancouver, Canada

${ }^{2}$ Department of Electrical Engineering and Computer Science, University of Michigan, Ann Arbor, USA

\section{ABSTRACT}

This paper reports a micromachined capacitive pressure sensor that does not use the traditional cavity and diaphragm, and its use in aqueous environment. The device is fabricated with two micromachined plates of stainless steel and an intermediate polymer layer that is soft enough to deform in the target pressure range. A polyurethane room-temperature-vulcanizing liquid rubber of $38-\mu \mathrm{m}$ thickness is used as the deformable material. For frequency-based interrogation of the capacitance, a passive inductor-capacitor tank is fabricated by combining the capacitive sensor with an inductive coil, which is formed using an $80-\mu$ m-diameter copper wire. Wireless sensing in liquid is demonstrated by monitoring the variation in the resonant frequency of the tank via an external coil that is magnetically coupled with the tank. The sensitivity at room temperature is measured to be $23-33 \mathrm{ppm} / \mathrm{KPa}$ over a dynamic range of $340 \mathrm{KPa}$, which is shown to match a theoretical estimate obtained by a bonded elastomer model. The geometrical impact on the frequency response is also evaluated.

\section{INTRODUCTION}

Capacitive pressure sensors are favored for low-power and telemetric applications since they draw no DC power, and conveniently form passive inductor-capacitor (L-C) tank circuits for frequency-based measurement of pressure [1-3]. Micromachined capacitive pressure sensors typically use an elastic diaphragm with fixed edges and a sealed cavity in between the diaphragm and the substrate below [4,5]. Since this configuration relies on the deflection of a relatively thin diaphragm against a sealed cavity, in some applications there is a concern of robustness of the diaphragm and leaks in the cavity seal. For example, in implantable applications, diaphragm or cavity failure is unacceptable. Survivability with high over-pressures and mechanical robustness are critical for certain military uses. Lead transfer for the sealed electrode has also been a persistent challenge.

This research explores a capacitive pressure sensor that consists of two micromachined metal plates with an intermediate polymer layer, eliminating the need of diaphragms and cavities. Use of polymeric material that is soft enough to deform over a target pressure range allows thickness of the polymer, or capacitance of the parallel plate capacitor, to be dependent on hydraulic pressure that surrounds the device (Fig. 1). This capacitive change can be interrogated by monitoring the resonant frequency of an L-C tank in which the sensor serves as a capacitor of the tank. The tank can be formed by coupling an inductor coil with the sensor separately, or it can be done by winding an insulated wire directly on the sensor [6]. The wireless interrogation can be implemented using an external antenna/inductor that is magnetically coupled with the L-C tank device (Fig. 2). The simple sandwich configuration of the pressure sensor provides not only mechanical robustness by eliminating the risk of cavity/diaphragm failures but also more freedom in the selection of structural materials. The latter feature will allow us to use appropriate materials to achieve an inherent compatibility of the device with target environments, e.g., those in corrosive liquid and the human body, circumventing packaging-related limitations that can negatively impact the cost and applicability of the device.
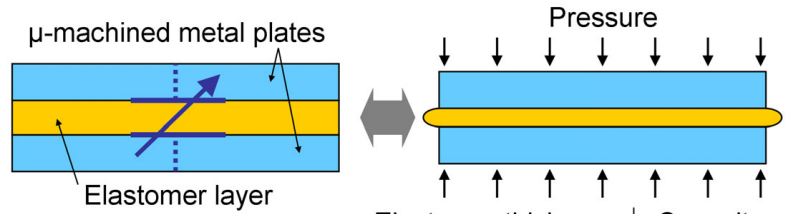

Elastomer thickness $\downarrow$, Capacitance $\uparrow$

Figure 1: Cross sectional view of the cavity-less bulk-metal/ elastomer capacitive pressure sensor.

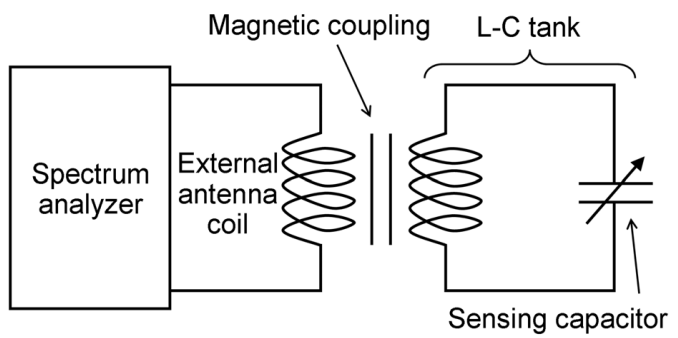

Figure 2: Electrical representation of the wireless measurement set-up for the $L$-C tank device.

\section{THEORY}

The capacitance of the device is determined by the thickness of the intermediate elastomer that is varied with the ambient pressure. For a rectangular layer of an incompressible, homogeneous elastomer that is bonded with rigid plates on both sides, the relationship between an applied pressure, $P$, on each of the plates and the resultant strain, $e$, can be expressed as [7]:

$$
P=\frac{E A}{2}\left(S^{2}-S_{0}{ }^{2}\right)-E\left[1+\frac{1}{3}\left(\frac{Y^{2}-W^{2}}{Y^{2}+W^{2}}\right)^{2}\right] \log (1-e)
$$

where $E$ is the Young's modulus of the elastomer, $2 Y$ and $2 W$ are the length and width of the rectangle layer, respectively. $A$ is a constant given by

$$
A=\frac{4}{3}+\frac{W}{Y}\left(2-\frac{11 W}{10 Y}\right)
$$

$S$ is a geometric parameter called shape factor, which is approximately represented for the structure by

$$
S=\frac{Y W}{2 T(Y+W)}=\frac{S_{0}}{(1-e)},
$$

where $2 T$ is the resultant thickness of the layer upon the compression and $S_{0}$ is the original shape factor with the initial thickness $\left(2 T_{0}\right)$ before the compression. The strain can be expressed as $e=1-T / T_{0}$. The final thickness determines the capacitance of the structure $C=\varepsilon(4 Y W) /(2 T)$, where $\varepsilon$ is the permittivity of the elastomer, and then the resonant frequency of the L-C tank, $f=1 /(2 \pi \sqrt{L C})$, where $L$ is the inductance of the tank. The permittivity of polyurethane is reported to be stable over the pressure range that is involved in this effort [8]. With these, the ratio of the resonant frequency after the compression to the original 
one and that for capacitance can be coupled with the strain as

$$
\left(\frac{f}{f_{0}}\right)^{2}=\frac{C_{0}}{C}=\frac{T}{T_{0}}=1-e
$$

where $C_{0}$ and $f_{0}$ are the original capacitance and resonant frequency prior to the compression, respectively. Therefore, the relationship between the applied pressure and the ratio in the resonant frequency, $f / f_{0}=F$, can be expressed using Eqs. (1) and (4) as

$$
P=\frac{E A S_{0}^{2}}{2}\left(\frac{1}{F^{4}}-1\right)-E\left[1+\frac{1}{3}\left(\frac{Y^{2}-W^{2}}{Y^{2}+W^{2}}\right)^{2}\right] \log \left(F^{2}\right)
$$

\section{FABRICATION}

Figure 3 illustrates the fabrication process for the sensors. The base and top plates with the indicated dimensions were cut from type-304 stainless steel sheets with thickness of $100 \mu \mathrm{m}$ and $50 \mu \mathrm{m}$, respectively, using micro-electro-discharge machining ( $\mu \mathrm{EDM})[9]$ (Fig. 4). The top plate was designed to be slightly smaller than the base plate ( $50-\mu \mathrm{m}$ offset from all sides of the base plate) to assist with the self-alignment of the two plates in the assembly step performed later. The base plate was still connected to the original foil with two tethers after the machining as shown in Fig. 4. A two-part polyurethane RTV liquid rubber (Poly 74-20, part-A: polyurethane pre-polymer, part-B: polyol, Polytek Development Co., PA, USA) with the softener (part-C: plasticizer) was used to form very soft ( $<20$ Shore $A$ ) and robust rubber. This effort used the formulation of parts $\mathrm{A}: \mathrm{B}: \mathrm{C}=1: 1: 1$, which provides rubber with the Young's modulus of $67 \mathrm{KPa}$ [6]. After applying the mixed solution to the upper surface of the base plate, the top plate was placed on it. The top plate was self-aligned to the base due to surface tension of the solution. After curing, the device was released from the original foil (Fig. 5a). The cured polyurethane had $\sim 38-\mu \mathrm{m}$ thickness (Fig. $5 b)$, providing the nominal capacitance of $6.0 \mathrm{pF}$. An inductive coil

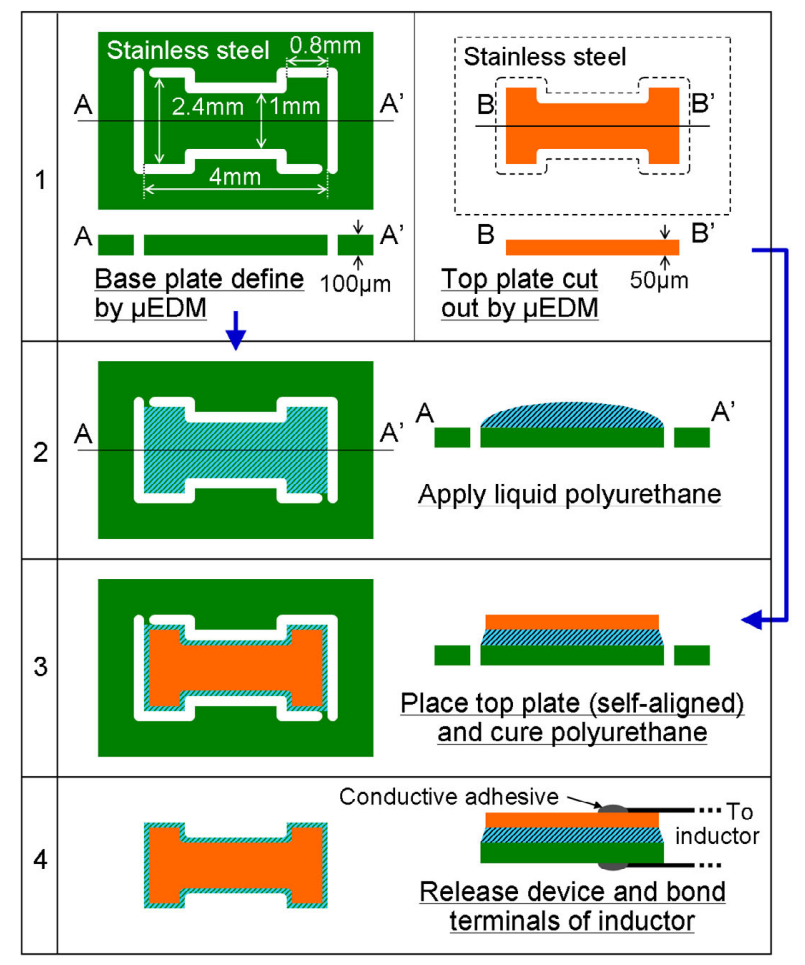

Figure 3: Fabrication process flow.

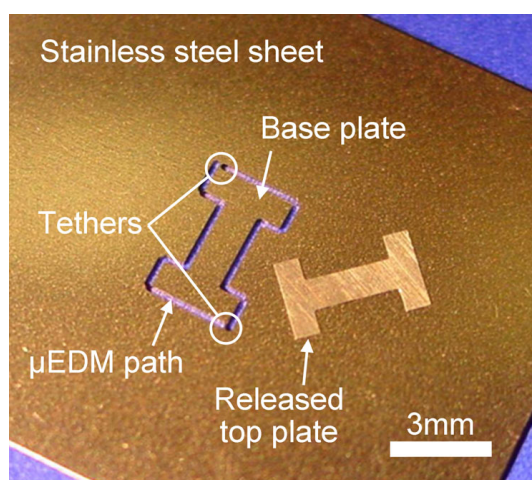

Figure 4:

Individual capacitive plates fabricated by $\mu$ EDM of type-304 stainless steel foil.
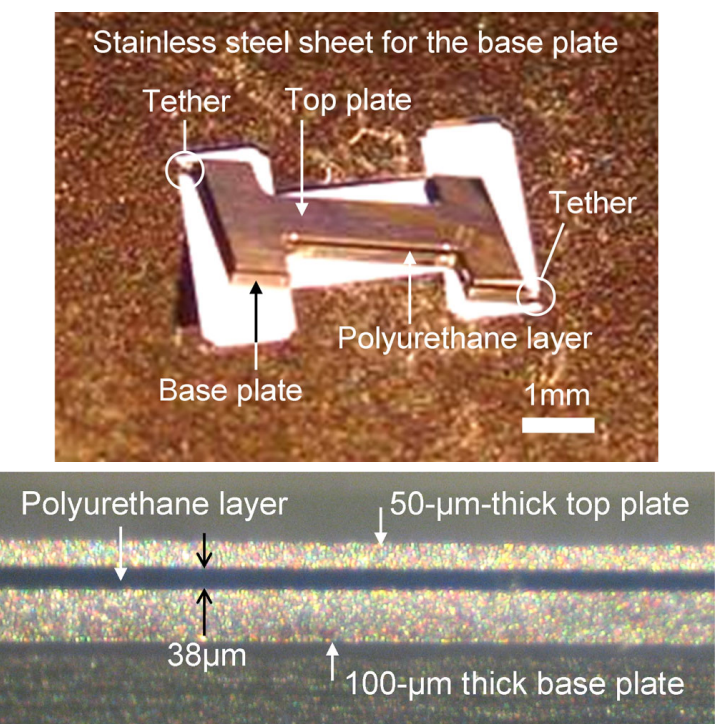

Figure 5: (a: upper) Fabricated device with the cured polyurethane being released from the original stainless-steel foil by breaking the tethers; (b: lower) sidewalls of the capacitive device showing stainless-steel/polyurethane layers.

(5-mm diameter, 5 turns) of 80- $\mu$ m-thick enamelled copper lead was coupled with the device to form an L-C tank by bonding the terminals of the coil to the capacitive plates with conductive adhesive. The oxide layers of the stainless steel were mechanically removed prior to the bonding to lower the contact resistance, i.e., increase the quality factor of the tank. The resonant frequency of the fabricated L-C tank was measured to be $95 \mathrm{MHz}$ in air, which is close to the theoretical value ( $\sim 80 \mathrm{MHz})$ obtained with the measured capacitance and inductance of the tank.

\section{EXPERIMENTAL RESULTS}

Figure 6 illustrates the set-up used for the wireless sensing tests. The fabricated L-C tank devices were placed within a sealed plastic chamber, and magnetically coupled with an external coil through the chamber walls. The resonant frequency of the tank was monitored by tracking the frequency of the characteristic peak, which was reflected by the resonance of the tank, in an s-parameter (s11) of the external coil that was connected to a network-spectrum analyzer while changing pressure inside the chamber. The chamber was filled with deionized water to demonstrate operation in liquid. The devices provided a distinct resonant peak, even without packaging/coating for electrical protection. With the same set-up, the frequency dependence on temperature was also evaluated at atmosphere pressure. Temperature of the chamber was controlled 


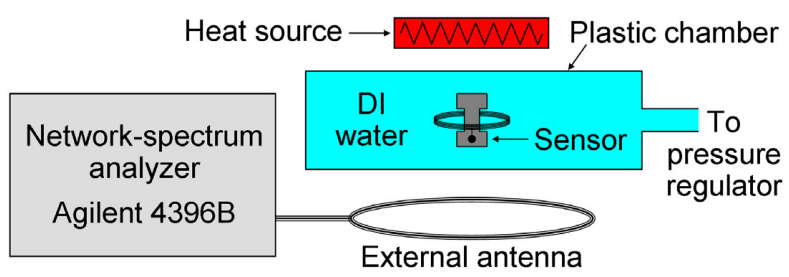

Figure 6: Set-up for wireless testing.

by changing the distance between the device and a source of heat located outside of the chamber as shown in Fig. 6.

Figure 7 shows a typical measured response with the wireless set-up at room temperature in DI water. Compared to the $95 \mathrm{MHz}$ resonant frequency in air, the reduced resonant frequency was expected with the increased parasitic capacitance due to the operation in water. The sensitivity is calculated to be 23-33 $\mathrm{ppm} / \mathrm{KPa}$ for the pressure range up to $340 \mathrm{KPa}$. The same measurement at $40{ }^{\circ} \mathrm{C}$ also plotted in Fig. 7 exhibits a similar curve with an offset of about $+0.4 \mathrm{MHz}$ from that at room temperature. The resonant frequency measured with varying temperature at atmosphere pressure is plotted in Fig. 8, indicating a linear dependence with its coefficient of $+783 \mathrm{ppm} /{ }^{\circ} \mathrm{C}$. The increase of the resonant frequency suggests the decrease of the capacitance, which can be due to the thermal expansion of the polyurethane. (The dielectric constant of polyurethane elastomer was reported to be stable at the temperature range used in this experiment [10].)

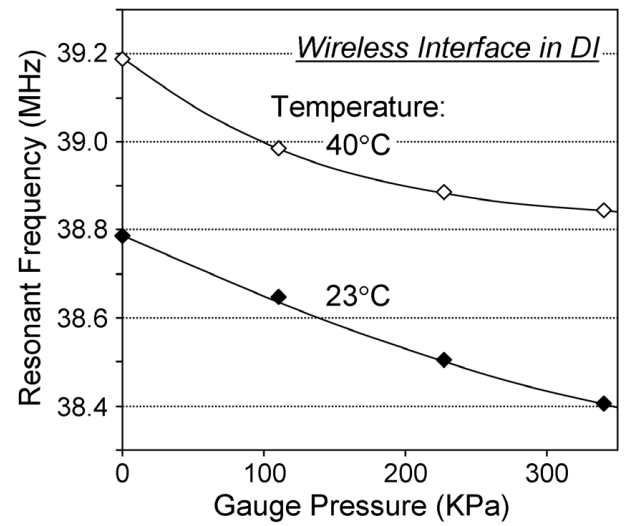

Figure 7: Typical frequency response of the fabricated device vs. pressure measured with the wireless set-up in Fig. 6 at room and elevated temperatures.

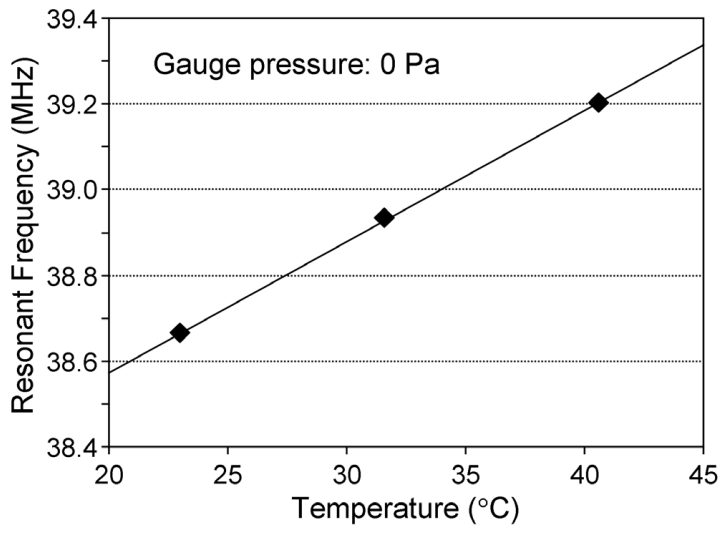

Figure 8: Typical frequency dependence on temperature measured at atmosphere pressure.

\section{THEORETICAL EVALUATION}

It is worth evaluating the measurement results obtained and their consistency with the theoretical estimation. To simplify the task for this initial analysis using Eq. (5), the following calculation assumes that the capacitive structure has a simple rectangular shape with $4 \times 1-\mathrm{mm}^{2}$ area, which corresponds to the largest rectangular portion of the actual design (Fig. 3). It further assumes that the dimensions of the top and base plates as well as the intermediate elastomer layer are all identical.

With the measured polyurethane thickness $2 T_{0}=38 \mu \mathrm{m}$ and the lateral dimensions of the selected rectangle, i.e., $2 Y=4 \mathrm{~mm}$ and $2 W=1 \mathrm{~mm}$, the constant $A$ and the shape factor $S_{0}$ are calculated to be 1.76 and 10.5, respectively. Using Eq. (5) with these values and $E=67 \mathrm{KPa}$, the normalized resonant frequency, $F$, as a function of hydrostatic pressure, $P$, is numerically solved and plotted in Fig. 9. The "band" shown in the graph represents the possible range of the theoretical response with an assumption of $\pm 5 \%$ variations in the thickness and Young's modulus of the polyurethane layer. The measurement result in Fig. 7 at room temperature is also plotted in the graph for comparison. It is clearly seen that the theoretical estimation matches well with the measured response at lower pressure. It can also be seen that the measured response deviates from the theoretical response as the pressure is increased.

Figure 10 shows calculated frequency change $(F)$ with pressure as a function of the width-to-thickness ratio $(W / T)$ of the rectangular

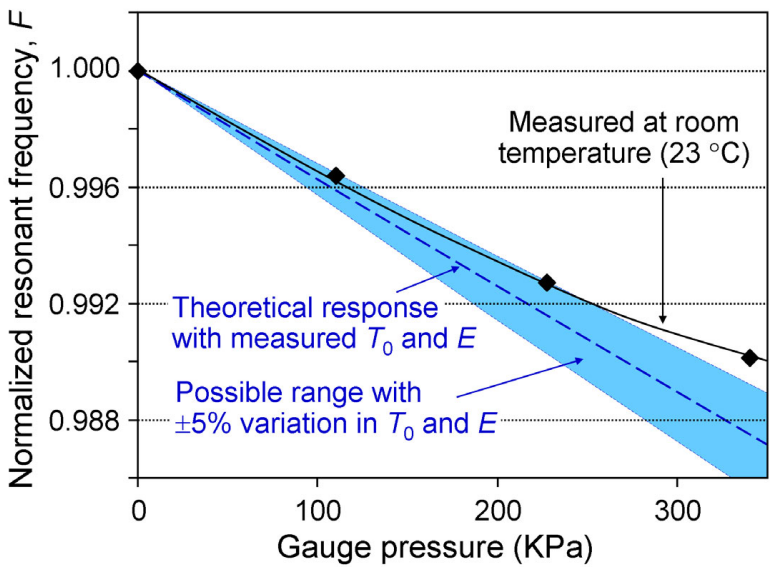

Figure 9: Comparison of relative frequency changes $(F)$ between the measured result from Fig. 7 and the theoretical response of the device with the simplified $4 \times 1-\mathrm{mm}^{2}$ rectangular shape.

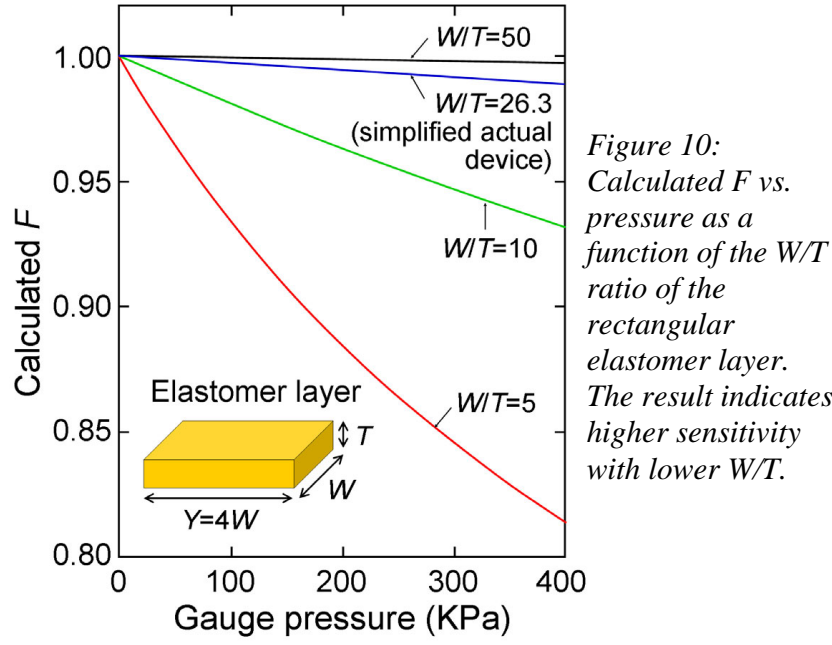


elastomer layer. The calculation assumes the length-to-width ratio $(Y / W)$ of 4 as in the simplified shape of the fabricated device used for the analysis in Fig. 9. The plot for $W / T=26.3$ corresponds to the actual device and identical to the theoretical plot in Fig 9. The result indicates the strong dependence of the sensor response on the $W / T$ ratio.

\section{DISCUSSION}

The slightly lower response and deviation from the theoretical estimation seen in Fig. 9 could be partially because of the presence of the extra portions (four $0.8 \times 0.7-\mathrm{mm}^{2}$ rectangles) in the actual device that were excluded in the analysis - these portions partially increase the width $(W)$, i.e., the $W / T$ ratio of the device, leading to a reduced response as predicted in Fig. 10. Another hypothesis may be related to the deformation of the capacitive plates especially in the thinner top plate. The compressive strain of the polymer layer depends on the lateral location on the structure under a uniform applied pressure [11]. Hence the upper plate can bend if it is not completely rigid, which is the real case. This deformation, i.e., non-uniform displacement of the plate, may also be a partial source of the deviation. Non-ideal factors attributed to thin layer of the polyurethane material including inhomogeneity of the material and inclusion of particles while mixing the liquid components of the material can be a potential contributor as well. Nevertheless, it is noteworthy that the theoretical model for the bonded elastomer, which was originally developed for macro-scale blocks, is useful to find an approximate response, within a limited pressure range (up to $300 \mathrm{KPa}$ ), of a micromachined device with an elastomer layer whose thickness is only a few tens of microns.

The device construction will need some optimization for improved performance and practicality of the device. The analytical result in Fig. 10 suggests a path to achieving higher sensitivity with modified designs of the device. The high temperature coefficient of this device may limit its applications. One of potential options to address the issue would be the use of composite rubbers that incorporate inorganic negative thermal expansion (NTE) nanoparticles [12]. The device will need to be coated with a dielectric layer for electrical insulation when the device is surrounded by a conductive medium. This will be important especially for biomedical and implant applications where the device makes direct contact with polarizable liquids such as body fluid and blood. These electrical and biological insulations can be easily achieved by the use of Parylene materials - the flexible, stretchable feature of the material is expected to minimize the impact of coating on the mechanical behavior of the device.

\section{CONCLUSIONS}

This research has explored a micromachined capacitive pressure sensor that eliminated both a diaphragm and a sealed cavity from its construction. The sensor consists of two metal plates and an intermediate polymer, which is expected to offer high mechanical robustness and reliability. The device was constructed with stainless-steel plates fabricated by a $\mu$ EDM technique and polyurethane liquid rubber as the polymer layer that permitted self-aligning of the micromachined plates in the assembly process. This material combination can offer good corrosion resistance and fracture toughness, potentially reducing the difficulties associated with packaging for selected applications. The sensor and a 5-mm-diameter copper coil were combined to form an L-C tank, which was successfully used to implement wireless frequency readout for pressure monitoring up to $340 \mathrm{KPa}$ gauge pressure in liquid. The maximum sensitivity was observed to be $33 \mathrm{ppm} / \mathrm{KPa}$. The comparison between the measured result and theoretical response obtained with a bonded elastomer model revealed the effectiveness of the model in predicting the sensor response.

\section{ACKNOWLEDGMENTS}

The authors would like to thank TRIUMF, Vancouver, B.C., Canada, for providing access to their measurement equipment and Mr. Mark Richardson at the University of Michigan, Ann Arbor, for his assistance in the micromachining work. Y. Gianchandani acknowledges partial support from the IR/D program of the National Science Foundation (NSF), USA. The findings do not necessarily reflect the views of the NSF.

\section{REFERENCES}

[1] A.D. DeHennis and K.D. Wise, “A Wireless Microsystem for the Remote Sensing of Pressure, Temperature, and Relative Humidity," IEEE/ASME J. Microelectromech. Syst., 14(1), 2005, pp. 12-22.

[2] K.H. Shina, C.R. Moona, T.H. Leeb, C.H. Limb, and Y.J. Kimb, "Flexible Wireless Pressure Sensor Module," Sensor. Actuator., A 123-124, 2005, pp. 30-35.

[3] M.A. Fonseca, J.M. English, M. von Arx, and M.G. Allen, "Wireless Micromachined Ceramic Pressure Sensor for High-Temperature Applications," IEEE/ASME J. Microelectromech. Syst., 11(4), 2002, pp. 337-343.

[4] H. Chau and K.D. Wise, "An Ultraminiature Solid-State Pressure Sensor for a Cardiovascular Catheter,” IEEE Trans. Electron Dev., 35(12), 1988, pp. 2355-2362.

[5] W.H. Ko and Q. Wang, "Touch Mode Capacitive Pressure Sensors for Industrial Applications,” Proc. IEEE Int. Conf. Micro Elec. Mech. Syst. (MEMS), 1997, pp. 284-289.

[6] K. Takahata and Y.B. Gianchandani, "A Micromachined Polyurethane/Stainless-Steel Capacitive Pressure Sensor Without Cavity and Diaphragm," Proc. IEEE Int. Conf. Solid-State Sensor. Actuator. Microsyst. (Transducers), 2005, pp. 483-486.

[7] J.M. Hill and A.I. Lee, "Large Elastic Compression of Finite Rectangular Blocks of Rubber," Q. J. Mech. Appl. Math., 42(2), 1989, pp. 267-287.

[8] Z.Y. Cheng, S. Gross, J. Su, and Q.M. Zhang, "Pressure-Temperature Study of Dielectric Relaxation of a Polyurethane Elastomer,” J. Polymer Sci. B Polymer Phys., 37(10), 1999, pp. 983-990.

[9] K. Takahata and Y.B. Gianchandani, "Batch Mode Micro-Electro-Discharge Machining," IEEE/ASME J. Microelectromech. Syst., 11(2), 2002, pp. 102-110.

[10] J. Su, Q.M. Zhang, C.H. Kim, R.Y. Ting, and R. Capps, "Effects of Transitional Phenomena on the Electric Field Induced Strain-Electrostrictive Response of a Segmented Polyurethane Elastomer,” J. Appl. Poly. Sci., 65(7), 1997, pp. 1363-1370.

[11] B.P. Holownia, “Compression of Bonded Rubber Blocks,” J. Strain Anal., 6(2), 1971, pp. 121-123.

[12] G. Agostini and F.G. Corvasce, "Tire with Low Thermal Expansion Component,” United States Patent Application No. 20070074801, 2007. 